\title{
Research on the Precision Measuring System of Steel Bearing Aperture Based on Machine Vision \\ LIU Xiao-jie ${ }^{1, a^{*}}$, WANG Tian-hu ${ }^{1, b}$, WANG Man-li, ${ }^{1, c}$ \\ ${ }^{1}$ Jiangsu University of Technology, Changzhou, Jiangsu Province 213001, P. R. China

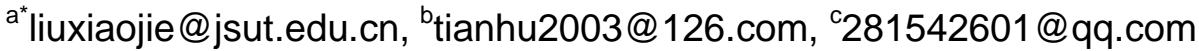

Keywords: Steel bearing aperture, Precision measuring system, Machine vision, Digital image processing

\begin{abstract}
A new measurement system was designed in order to improve the accuracy of the non-contact type high-precision measurement of Steel bearing aperture. Firstly, the median filter preprocessing algorithm was designed to remove the impulse and salt-pepper noise in the image, and the edge details of the image was also preserved. Secondly, the image processing equalization algorithm was applied to improve the image information contrast, which the gray level of original image has been extended to the entire gray level range of the output image. Thirdly, the improved canny edge detection algorithm was applied to detect edge more accurately, which employed the convolution operation of the smoothing filter impulse response of first order differential and the original image. The noise suppression and robustness of the operator were improved. Finally, the contour image of the measured part was filtrated to calculate its size by the aperture image detection algorithm. Its system algorithm software was designed based on the machine vision and computer control technology, which used the Visual $\mathrm{C}++$ development language in the integrated development environment of OpenCV. The results show that the precision measuring system is highly accurate and efficient for the parts size measurements. It is helpful to improve the accuracy of the Precision Manufacturing Technology and Measurements.
\end{abstract}

\section{Introduction}

In modern machinery manufacturing industry, technologies of precision manufacturing and measurements are fundamentally important [1-3]. They can ensure the quality of products [4-6]. For the current manufacturing industry, detection techniques have become more and more sophisticated since consumers increasingly demand high quality and diversity of products. [7-9]. Immediacy and non-contact are the two characteristics of modern detection techniques. Therefore, our system introduces machine vision in the field of industrial inspection [10-12]. Our experiments intend to find a detection technique which is rapid, non-contact, automatic and accurate.

\section{General Block Diagram of the System}

Visual detection system can measure parts by non-contact with Visual C++ language. And it realizes the function of detection with high efficiency and high precision measurement. Hardware system mainly consists of LED lighting system, CCD camera, image acquisition card and so on. CCD camera collects the image of parts. Then image acquisition card transmits images to a computer for processing. The system applies software based on the idea of modular design with OpenCV and Visual C++ language. General block diagram of the system is shown in Fig. 1. 


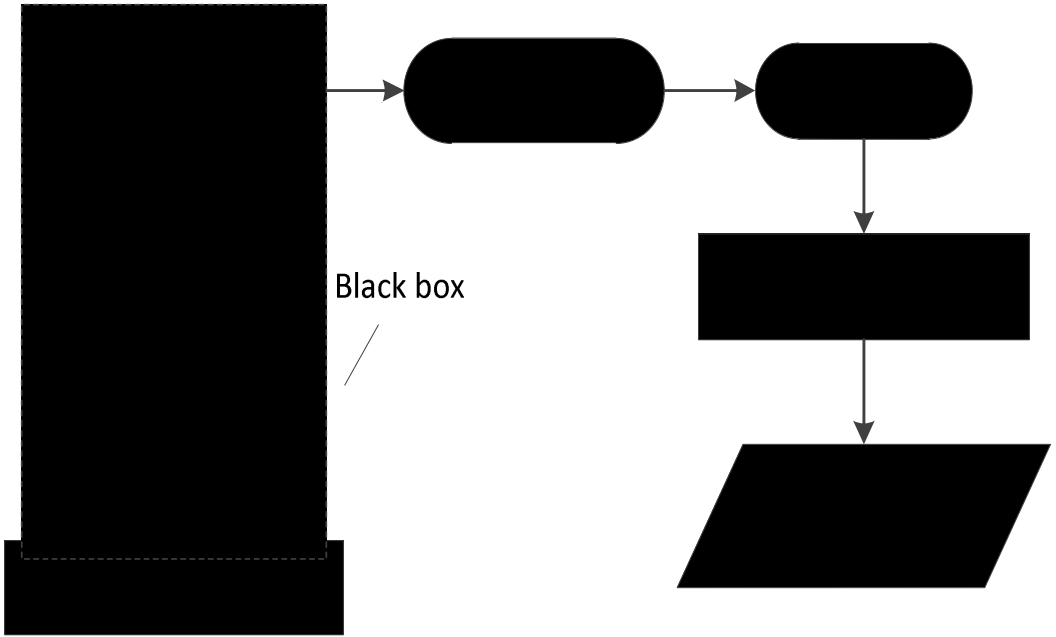

Fig. 1 General block diagram of the system.

\section{Visual Image Information Processing}

Image Preprocessing. Image pre-processing employs the method of median filter with nonlinear filtering. The meaning of median filter: firstly, selecting a pixel, all pixels value in this eight neighborhood order from small to large in accordance with the arrangement. And then it takes the value instead of the gray value of the origin value.

With the order from large to small as follows:

$$
\mathrm{x}=\operatorname{med}\left(x_{1}, x_{2}, x_{3} \ldots x_{n}\right)=\left\{\begin{array}{c}
x_{(n+1) / 2}^{\prime}, \text { nodd } \\
\frac{1}{2}\left(x_{n / 2}^{\prime}+x_{n / 2+1}^{\prime}\right), \text { neven }
\end{array}\right.
$$

If the input of image is $f(x, y)$, the output of image with the handling of median filter is $g(x, y)$ :

$$
g(x, y)=\operatorname{med}[f(i, j)],(i, j) \in A
$$

In the Eq. 2, A: $-(\mathrm{i}, \mathrm{j})$ as the center of all the values of the neighborhood. The histogram of original image is shown in Fig. 2. Impression image with median filter is shown in Fig. 3. From all of them, median filter can deal with noise perfectly. At the same time, it holds the detail of edge. And then it makes the image become gentle by histogram equalization which is shown in Fig. 4 . From it, we finds that treatments make the value of original image expand in the entire gray level range.

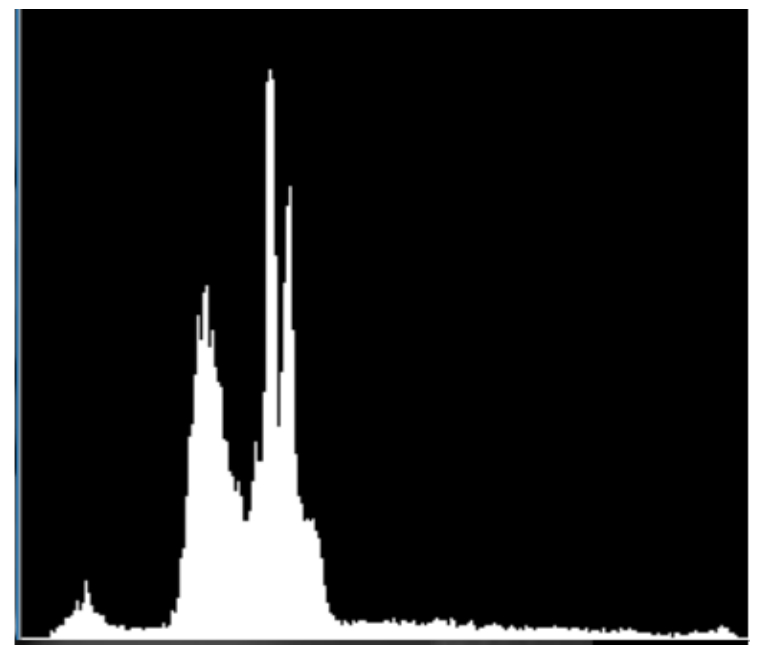

Fig. 2 Original image. 


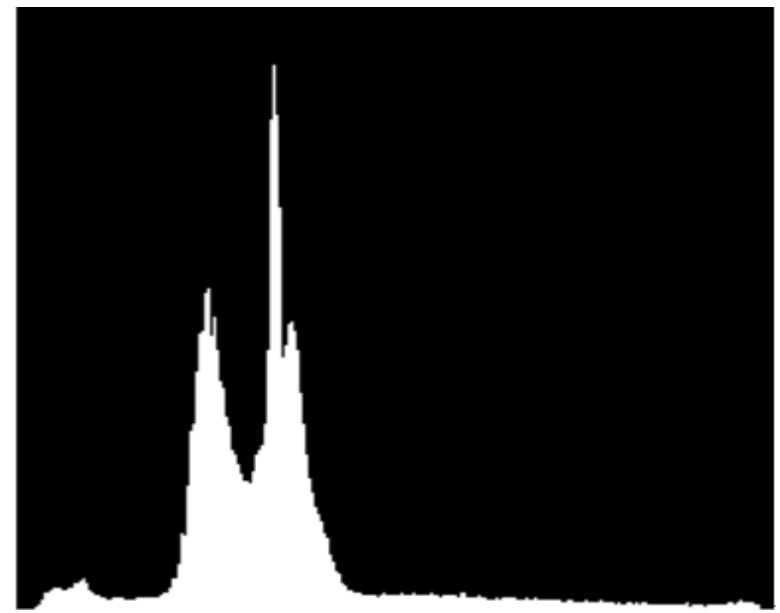

Fig. 3 Median filter.

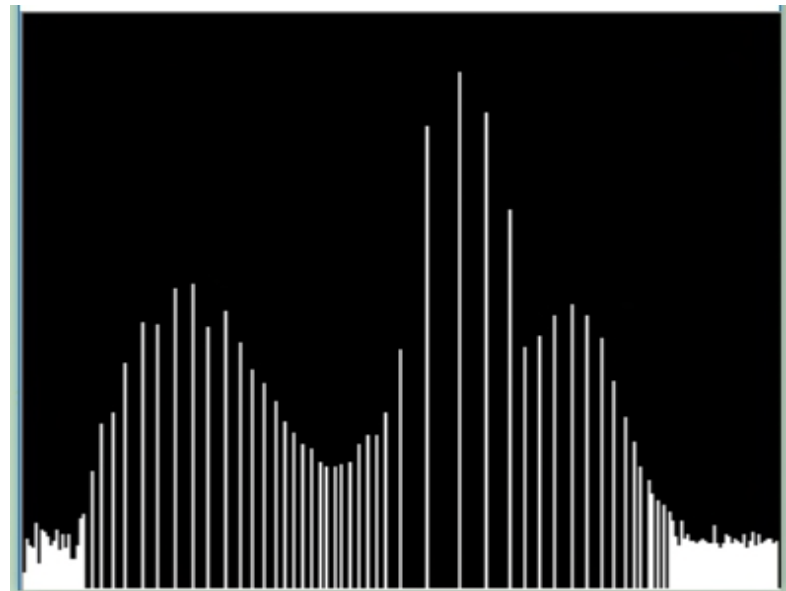

Fig. 4 Equalization.

Improved Canny Edge Detection. The improved canny operator uses a $3 \times 3$ neighborhood, which takes the possible influence of the pixels into account in every direction. The gradient $A(i, j)$ and the gradient direction $\theta(\mathrm{i}, \mathrm{j})$ of the neighborhood of the center point is as follows:

$$
\begin{aligned}
& \mathrm{A}(\mathrm{i}, \mathrm{j})=\operatorname{sqrt}\left(G_{x}^{2}(\mathrm{i}, \mathrm{j})+G_{y}^{2}(\mathrm{i}, \mathrm{j})+G_{45^{\circ}}^{2}(\mathrm{i}, \mathrm{j})+G_{135^{\circ}}^{2}(\mathrm{i}, \mathrm{j})\right) \\
& \theta(\mathrm{i}, \mathrm{j})=\tan ^{-1}\left(\frac{G_{y}^{2}(\mathrm{i}, \mathrm{j})}{G_{x}^{2}(\mathrm{i}, \mathrm{j})}\right)
\end{aligned}
$$

Formula (3) describes the edge intensity of the image; formula (4) describes the edge direction. It can be seen from the figure that the improved Canny operator can detect edge more clearly and accurately. It is shown in Fig. 5.

\section{Measurement and Determination of the Parameters of the Aperture Detection}

Although there are more than one edges for a machine part, only one of the edges need measuring. Hence it needs to select the specified edge of all the edges. Since the edge of the part is a circle, our experiment uses the method of Hough circle transformation to screen out the edge to be measured. The impression image of original image is shown in Fig. 6. 


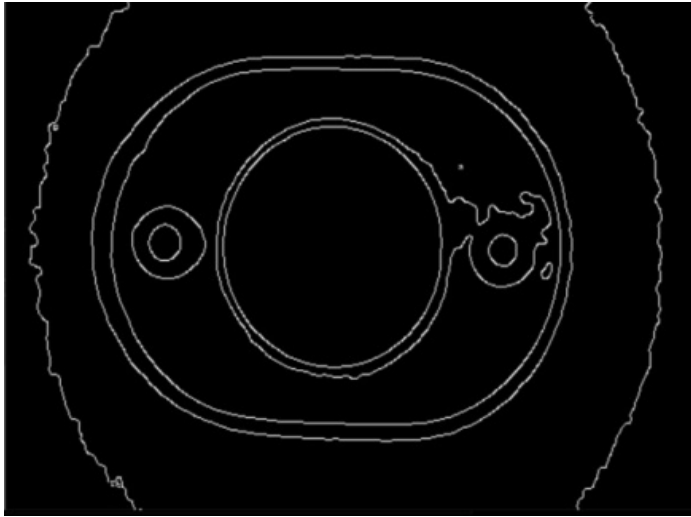

Fig. 5 Improved Canny detection.

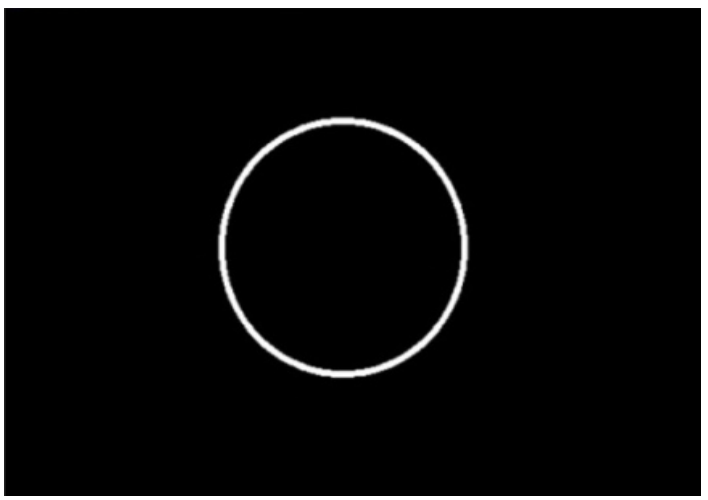

Fig. 6 Aperture detection.

\section{Experimental Result}

Materials. 1) With an aperture diameter of the standard steel material part as the measuring objects, the $2 / 3$ inch CCD camera was used in this system. 2) With the grade- 0 gauge block is used in the system calibration and each pixel size corresponding to $0.00564 \mathrm{~mm}$. 3) With Three-Coordinate Measuring Machine(UK LK-G90CS15.10.8, Precision:0.002mm, time consuming:22minutes), the standard steel material sample was measured as the standard diameter $25.011 \mathrm{~mm}$, which corresponds to the image pixel diameter contains 26562 pixels.

Methods. The standard steel material part in the different position of the conveyer belt was measured in this system, which the 10 times data were recorded and the results were shown in Table 1. After analyzation and calculation, we can obtain the parameter of part for average, variance, standard deviation, dispersion and so on. The results were shown in Table 2.

Table 1. Experimental measurement results

\begin{tabular}{|c|c|c|c|}
\hline Number & Bore diameter $/ \mathrm{mm}$ & Absolute error $/ \mathrm{mm}$ & $\begin{array}{c}\text { Relative } \\
\text { error \% }\end{array}$ \\
\hline 1 & 25.0137 & 0.0027 & 0.01079525 \\
\hline 2 & 25.0142 & 0.0032 & 0.01279437 \\
\hline 3 & 25.0170 & 0.0060 & 0.023989445 \\
\hline 4 & 25.0163 & 0.0053 & 0.021190676 \\
\hline 5 & 25.0135 & 0.0025 & 0.009995602 \\
\hline 6 & 25.0156 & 0.0046 & 0.018391908 \\
\hline 7 & 25.0145 & 0.0035 & 0.013993843 \\
\hline 8 & 25.0174 & 0.0064 & 0.025588741 \\
\hline 9 & 25.0178 & 0.0068 & 0.027188037 \\
\hline 10 & 25.0186 & 0.0076 & 0.03038663 \\
\hline
\end{tabular}


Table 2. Measurement parameter analysis

\begin{tabular}{|c|c|c|c|c|c|c|}
\hline & Max $/ \mathrm{mm}$ & Min $/ \mathrm{mm}$ & Aver $/ \mathrm{mm}$ & Variance $/ \mathbf{m m}^{\mathbf{2}}$ & $\begin{array}{c}\text { Standard } \\
\text { deviation } / \mathrm{mm}\end{array}$ & $\begin{array}{c}\text { Dispersion } \\
\text { degree } / \mathrm{mm}\end{array}$ \\
\hline Diameter & 25.0186 & 25.0135 & 25.01586 & $3.3382 \times 10^{-6}$ & 0.001827080 & 0.0051 \\
\hline
\end{tabular}

Based on the above measurement results, it can be known that the parts position is different among ten times measurements. The experiment shows that the error between test results and the standard size of parts can be strictly controlled within $10 \mu \mathrm{m}$. The result perfectly reaches our experimental expectation

\section{Conclusions}

As the image contrast near the boundary is different, we propose a measurement method based on image processing, which can find the location of boundary, and carry out the measurement of the aperture. This high precision measurement system is designed to realize the non-contact measurement for the steel bearing aperture, which used the Charge-coupled Device (CCD) as image sensor, and combined with the computer as powerful data processing ability. Experimental result shows that the measurement system can control the diameter error within $10 \mu \mathrm{m}$. It proves that the system meets the requirements of parts size precision measurement and it can also be applied to measure other aperture of smaller parts with non-contact measurement. Therefore this system provides a reference for the similar devices.

\section{Acknowledgments}

The authors would like to thank the funding support from Changzhou science and technology project (industry) of China (CE20150014) and the Natural Science Foundation of Jiangsu Province (No. BK20150247).

\section{References}

[1] F. Keceki, H. H. Nagel, Machine vision based estimation of pose and size parameters from a genetic work piece description, IEEE T. Robot. Autom. 3 (2001) 2159-2164.

[2] S. Sirkis, T. J. Lim, Displacement and Strain Measurement with automated Grid Methods, Experimented Mechan. 31(4) (1991) 382-388.

[3] C. Harris, Geometry from visual motion, Active Motion, MITpress, Cambridge. (1992) 263-284.

[4] F. Heijden, Image based measurement system, John Wiley \& Sons, (1995) 25-39.

[5] T. S. Newman, A. K. Jain, A survey of automated visual inspection, Comput. Vis. Image Understand. 61(2) (1995) 231-262.

[6] T. Lindeberg, Edge detection and ridge detection with automatic scale selection, Comput. Vis. Pattern Recognit. Los A Lamitors: IEEE, (1996) 465-470.

[7] J. L. Krahe, P. Pousset, The detection of parallel straight lines with the application of the Hough transform, Pattern Recognit. 11 (1998) 939-941.

[8] A. A. Hashim, P. E. Clement, Computer vision in a manufacturing process, Proceed. Robot Vis. (1994) 417-427.

[9] H. AlDmour, A. AlAni, Quality optimized medical image information hiding algorithm that employs edge detection and data coding, Comput. Method. programs Biomed. 127 (2016) 24-43.

[10]A. Mayur, R. Girish, Respiratory Mask Selection and Leakage Detection System Based on Canny Edge Detection Operator, Procedia Comput. Sci. 78 (2016) 323-329. 
[11]Z. Qu, X. Fang, H. Su, Measurements for displacement and deformation at high temperature by using edge detection of digital image, Appl. Opt. 54(29) (2015) 8731-8737.

[12]H. Choi, D. N. Wadduwage, T. Y. Tu, Three-dimensional image cytometer based on widefield structured light microscopy and high-speed remote depth scanning, Cytometry Part A 87(1) (2015) 49-60. 